Henning Klauß

\title{
RDA: Folgen für die Katalogisierung und die OPAC-Gestaltung
}

Zusammenfassung: Das Regelwerk RDA wird einige Veränderungen für den Katalog von Bibliotheken erbringen, die für Benutzer hilfreich sind. Dieser Fortschritt wird auch für die Katalogisierer und die Gestaltung des OPACs Fortentwicklungen erzwingen. Hierfür muss die in Bibliotheken verwendete Software sowohl hinsichtlich Katalogisierung wie OPAC fortentwickelt werden, was anhand von Beispielen gezeigt wird.

Schlüsselwörter: RDA, Katalogisierung, OPAC

\section{RDA: Consequences for the cataloguing and the OPAC formation}

Abstract: The catalog code RDA will furnish some changes for the catalog of libraries, that are helpful for users. This progress will force also for the cataloguer and the formation, the design of the OPACs further development. For this purpose, the library software must both be developed regarding cataloguing such as OPAC, what is shown by means of examples.

Keywords: RDA, cataloguing, OPAC

Henning Klauß: klauss@europa-uni.de

Der folgende Aufsatz ${ }^{1}$ bezieht sich auf die Bibliothekssoftware SISIS von OCLC das hier Dargestellte ist aber mit bestimmten Modifizierungen auch für andere Bibliothekssoftware relevant.

Das Regelwerk RDA (Resource Description and Access) soll nach derzeitigem Stand in deutscher Übersetzung erst Ende 2013 fertig sein und für Verbünde 2015 verbindlich werden - vorerst gibt es, abgesehen von Vorreitern wie der DNB, nur Planungen, Entwürfe und Zwischenstände. Insofern dürfen Sie nicht erwarten, zum jetzigen Zeitpunkt alles dargestellt zu bekommen, was für die Gestaltung von

1 Der Aufsatz geht zurück auf ein auf dem 28. SISIS-Anwenderforum Berlin Brandenburg (7.11.2012) gehaltenen Vortrag. 
SISIS bzgl. RDA irgendwann einmal relevant sein wird. Ich kann noch mit keiner Detailliste aufwarten, es ist mir aber wichtig, dass der Blick von OCLC rechtzeitig auf künftige, durch RDA bestimmte, Erwartungen an OCLC gerichtet wird. Irgendetwas ist immer zu spät, aber wenn es die Möglichkeit gibt, erwartbare Probleme durch rechtzeitiges Reagieren kleiner zu halten, sollte man das tun - ich will das mit diesem Aufsatz versuchen.

Bibliothekssoftware entwickelt sich u. a. im Hinblick auf die für die Katalogisierung gültigen Regelwerke. Die können sich ändern. In Deutschland gilt demnächst statt RAK RDA, wodurch der Komfort des Katalognutzers verbessert werden soll². Dieser Regelwerkswechsel erzwingt einige Innovationen.

RDA arbeitet mit Entitäten. So gibt es:

- Werk (intellektuelle/künstlerische Schöpfung)

- Expression (intellektuelle/künstlerische Realisierung eines Werkes: Auflage, Übersetzung ...)

- Manifestation (physische Verkörperung einer Expression: Mikrofiche statt Papier, anderer Verlag ...)

- Exemplar (einzelnes Stück einer Manifestation).

Ferner gibt es in RDA Beziehungen, z. B. „ist Zusammenfassung von“, „Teil-Ganzes“ (beispielsweise „Schriftenreihe - Stücktitel“), zu Vorgängern und Nachfolgern.

Es ist notwendig, dass SISIS in der Lage ist, alle diese Entitäten und Beziehungen vollständig abzubilden und in einer Weise zu verknüpfen, die den Schritt in jede Richtung ermöglicht. - Es darf hierbei also keine Limitierungen geben: Weder die Anzahl der Verknüpfungsmöglichkeiten noch die Richtungen dürfen beschränkt sein.

$\mathrm{Zu}$ einem Teil dieser Anforderungen, die durch RDA notwendig werden, ist SISIS schon heute in der Lage. Einer Reihe von Anforderungen genügt SISIS noch nicht - hier sind Fortentwicklungen notwendig.

Ich will hier nicht jedwede laut RDA mögliche Verknüpfung besprechen, nur an Beispielen zeigen, was künftig in SISIS realisiert werden muss. Das sei exemplarisch an einigen Relationen zwischen Expressionen gezeigt:

RDA verknüpft Titelaufnahmen mit denen zu Vorgängern und Nachfolgern von Zeitschriften, zu anderen Auflagen von Büchern und anderen Ausgaben, wie Übersetzungen oder andere physische Formate.

2 RDA soll auch in Archiven, Museen, Verlagen usw. Verwendung finden. 
Bisher kann ein Benutzer auf all das nur in den Fußnoten einer Titelaufnahme hingewiesen werden - er muss, wenn er eine solche Titelaufnahme sucht, in die Mehrfachtrefferliste zurückgehen oder eine neue Recherche absetzen.

Die Bewegung von einer Titelaufnahme zu einer anderen Titelaufnahme, die laut RDA zusammenhängen, sollte meines Erachtens so gestaltet werden, dass dieser Zusammenhang für Mitarbeiter arbeitsökonomisch herzustellen und für Benutzer leicht nachzuvollziehen sind. Ich fände es daher sinnvoll, wenn dieser Übergang von einer Titelaufnahme zur nächsten vom System generiert werden würde und ein Benutzer einen Vorschlag, z. B. eine Zeichenkette nur noch anklicken muss.

Derzeit geht letzteres nicht bzw. nicht vollständig, da in SISIS zur Zeit nur folgende Verknüpfungsmöglichkeiten gegeben sind:

1. Band bzw. Stücktitel und ÜG

2. Titelaufnahmen zur GND (Personen, Schlagwörter, Körperschaften)

3. Titelaufnahmen zu sonstigen Normdateien, z. B. Notationen

4. Titelaufnahmen zum Ausleihsystem

5. Titelaufnahmen zum Erwerbungssystem

6. Aufsatz-Quelle

OPAC-Recherchen satteln hierauf auf: Es gibt u. a. den sog. „Quickseach“ - das sind im OPAC die unterstrichenen, blauen Felder, um Titelaufnahmen, die mit diesem Namen eines Autoren, diesem Schlagwort usw. verknüpft sind, zu ermitteln: Es können bis zu zehn Felder in den Quicksearch aufgenommen werden, so dass ein Benutzer nur den Namen eines Autoren, einer Körperschaft oder ein Schlagwort anklicken muss und dann eine Liste erhält, was ansonsten von diesem Autoren vorliegt oder mit diesem Schlagwort verknüpft ist.

Verknüpfungen zu anderen Ausgaben, wie z. B. Online-Ausgaben, zu Vorgängern und Nachfolgern, auf die per „Später u.d. T. ...“, „Früher u. d. T. ...“ oder „Vorgänger“ hingewiesen wird, auf Zusammenhänge wie „darin aufgegangen“ usw. können derzeit nicht in SISIS gemacht werden. Es gibt nur den textlichen Hinweis auf Vorgänger und Nachfolger, nicht aber die Möglichkeit, darauf per Klick zuzugreifen. ${ }^{3}$

3 In ALEPH geht das bereits: Es heißt dort „Parlink“. Das funktioniert in ALEPH mittels IDs. Den Rückgriff auf die ID finde ich naheliegend. Das setzt natürlich voraus, dass klar ist, welche IDs verwendet werden: Die lokalen IDs, die Verbund-IDs oder ggf. die ZDB-IDs - aber wenn die für RDA notwendigen Verknüpfungen in SISIS anders gemacht werden, ist das auch ok - Hauptsache, es funktioniert. 
Schön wäre es natürlich, wenn es diese Möglichkeit in SISIS geben würde. Die Verbund-IDs von Vorgängern und Nachfolgern werden in den Titelaufnahmen protokolliert (bei Anschluss an den B3Kat in den 1500-Feldern von SISIS), aber derzeit nicht lokal weiter genutzt. Sinnvoll wäre es, diese Daten fruchtbar zu machen, indem Vorgänger und Nachfolger angezeigt und anklickbar gemacht werden würden. ${ }^{4}$

Dass Verknüpfungen zu Vorgängern und Nachfolgern, anderen Ausgaben etc. in SISIS derzeit gar nicht gemacht werden können, ist bedauerlich; die Verknüpfungen via Quicklink sind an dieser Stelle schon mal ein guter Anfang, der aber verbesserungsbedürftig ist.

Derzeit sind, ich wiederhole das, max. zehn Felder für die sog. Quicksearch zu wählen. Das reicht meines Erachtens nicht aus: Wenn für einen Sachverhalt ein einziges (multiples) Feld genutzt wird, ist dafür nur eine der zehn Möglichkeiten fällig; wenn zwei Felder genutzt werden, sind zwei der zehn Möglichkeiten fällig usw. Es wäre aber sinnvoll, wesentlich mehr Möglichkeiten zu schaffen, denn für nur für einen einzigen Sachverhalt müssen manchmal eine Reihe von Feldern verknüpft werden:

- Personennamen machen die Verknüpfung mit den Feldern für Verfasser, SBP, Interpret, Gefeierte Person erforderlich. (in SIKIS die Felder 100, 101, 102, 103, 1800).

- Schlagwörter machen je nach Sprache die Verknüpfung mit mehreren Feldern nötig (deutsch, englisch, polnisch, französisch - je nach Feldstrukturtabelle werden allein für die subject headings bis zu acht Felder benötigt).

- Körperschaften machen die Verknüpfung mit den Feldern „Urheber“ und mit „Beteiligte Körperschaft“ erforderlich.

- Notationen: Je nachdem, welche Klassifikationen die Bibliothek anbietet.

Inhaltserschließung, also die Vergabe von Notationen oder Schlagwörtern, wird nicht von jeder Bibliothek in gleicher Ausführlichkeit durchgeführt, aber die meisten Titelaufnahmen werden importiert und dann liegen in den Bibliothekskatalogen bei vielen Titelaufnahmen verschiedene Schlagwörter, subject headings, Notationen von der Universalen Dezimal-Klassifikation (UDK), der Dewey Decimal Classification (DDC), der Regensburger Verbundklassifika-

4 Die Felder mit den Verbund-IDs können in SISIS zur Anzeige im OPAC gebracht werden, aber davon hat gewiss kein Benutzer etwas. Diese IDs können derzeit von Titelaufnehmern genutzt werden, um Verknüpfungen manuell aufzubauen - wünschenswert aber wäre der automatische Aufbau einer Verknüpfung. 
tion (RVK) usw. vor. Und der OPAC sollte ja nicht nur das für Benutzer fruchtbar machen, was die eigene Bibliothek eingegeben hat, sondern alles, was da ist, egal ob in Eigenproduktion erzeugt oder importiert. Unabhängig von RDA, aber dann erst Recht: Die Zahl der möglichen Quicklinks muss erhöht werden.

Das kann selbstverständlich und gerne auch auf die wesentlich elegantere Art gelöst werden, indem alle über die FSTAB verknüpften Felder automatisch berücksichtigt werden.

Sonstige RDA-Verknüpfungen müssen praxistauglich hergestellt werden. Zur Praxistauglichkeit gehört: Für Bibliotheken, die in einem Verbund sind, kann eine Titelaufnahme z. B. den Hinweis auf Vorgänger und Nachfolger enthalten. Aber es gibt Bibliotheken, die entweder den Vorgänger oder den Nachfolger oder beides nicht haben. Ein Mitarbeiter, der im Verbund katalogisiert, darf nicht gezwungen sein, sich darum zu kümmern, ob eine Titelaufnahme in den Katalog einer Bibliothek gespielt wird, die den Vorgänger oder Nachfolger hat oder nicht hat: Eine Titelaufnahme im Verbundkatalog muss in verschiedenen Bibliothekskatalogen entsprechend den lokalen Bedingungen unterschiedlich aussehen, es muss beim Datenimport in die Lokalsysteme automatisch dafür gesorgt werden, dass ggf. zwei oder evtl. auch drei partiell verschiedene Titelaufnahmen daraus werden: Manche Bibliotheken haben Vorgänger und Nachfolger, manche nur Vorgänger, andere nur Nachfolger. - Derzeit entfällt die Notwendigkeit einer Zwei- oder Dreiteilung, weil man nichts anklicken kann: Es gibt nur den textlichen Hinweis auf Vorgänger und Nachfolger. Wenn man da hoffentlich demnächst weitermachen kann, indem man anklickt, ergibt sich die Notwendigkeit einer Zwei- oder Dreiteilung der Titelaufnahmen.

\section{Zudem}

- Umlenkungen müssen berücksichtigt werden,

- die vorzunehmenden Modifikationen müssen für alle SISIS-Bibliotheken fruchtbar sein, egal, ob die InfoGuide, TouchPoint oder nur den WWWOPAC nutzen.

Es geht mir hier nicht um Vollständigkeit. Es geht mir nur darum, dass der Blick von OCLC rechtzeitig auf künftige, durch RDA bestimmte Erwartungen an OCLC gerichtet wird. Es ist ein verbreitetes Spiel der Softwarebranche, Bananen beim Endverbraucher reifen zu lassen, d. h. die Verbraucher testen zu lassen. Die Endverbraucher sind hier die Bibliotheken. Und wir wollen möglichst ausgereifte, durchgetestete, den dann gültigen Anforderungen genügende Produkte haben.

Fazit: Jemand von OCLC muss sich in RDA einarbeiten, sich über Fortentwicklungen informieren und infolgedessen mögliche und/oder erforderliche Produktentwicklungslinien aufzeigen, die dann in SISIS realisiert werden. 
Da OCLC aus den USA kommt und dort u. a. bzgl. RDA manches schneller geht, kann dort bestimmt gelernt oder abgekupfert werden.

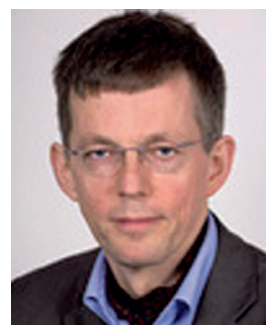

Dr. Henning Klauß, Stellvert. Direktor der Universitätsbibliothek Europa-Universität Viadrina Frankfurt (Oder) Große Scharrnstraße 59

15230 Frankfurt (Oder)

Tel.: +49(0)335-55343397

Email: klauss@europa-uni.de 